\title{
MANIN: Multi-Attention Neural Interaction Networks for Social Rumor Detection
}

\section{Sun Jiehu}

Xihua University

Wu Yue ( $D$ wuyue@mail.xhu.edu.cn)

Xihua University

\section{Research Article}

Keywords: Social networks, Attention mechanisms, Rumor detection, Co-attention

Posted Date: September 24th, 2021

DOl: https://doi.org/10.21203/rs.3.rs-888834/v1

License: (c) (i) This work is licensed under a Creative Commons Attribution 4.0 International License. Read Full License 


\title{
MANIN: Multi-Attention Neural Interaction Networks for Social Rumor Detection
}

\author{
Jiehu Sun ${ }^{1}$. Yue Wu ${ }^{1 *}$ \\ ${ }^{1}$ School of Computer and Software Engineering, Xihua \\ University, Chengdu 610039, China \\ *wuyue@mail.xhu.edu.cn
}

Received: date / Accepted: date

\begin{abstract}
With the fast-changing development of emerging online media, it has become apparent that information on social networks is characterized by extensive, fast and timely spreading. The absence of effective detection methods and monitoring means has led to a massive outbreak of rumors. Therefore, accurate detection and timely suppression of rumors in social networks is a vital task in maintaining social security and purifying public networks. Most existing work relies only on monotonous textual content and shallow semantic information, and lacks critical attention to and potential mining of user relationships. Such being the case, we can better improve these problems by employing attention mechanisms. In this paper, we proposea Multi-Attention Neural Interaction Network (MANIN) for rumor detection, which consists mainly of a self-attention-based BERT encoder, a post-comment coattention mechanism, and a graph attention neural network for mining potential user interactions. We have conducted numerous experiments on real datasets and verified their validity, and the results show that the model proposed by us outperforms existing models with an accuracy rate of $81.6 \%$.
\end{abstract}

Keywords Social networks · Attention mechanisms · Rumor detection · Co-attention

Mathematics Subject Classification (2020) MSC code $1 \cdot$ MSC code $2 \cdot$ more

\section{Introduction}

With the gradual rise of social media in recent years, it has become an important source for people to communicate with each other, express themselves and obtain information. Around 5\% of Facebook's monthly active users across the world are known to be fake accounts(Aaron Greenspan, "Reality Check"). Facebook officials said it had disabled more than 3.2 billion fake accounts between April 2019 and September 2019, with the number of duplicate accounts estimated at more than 255 


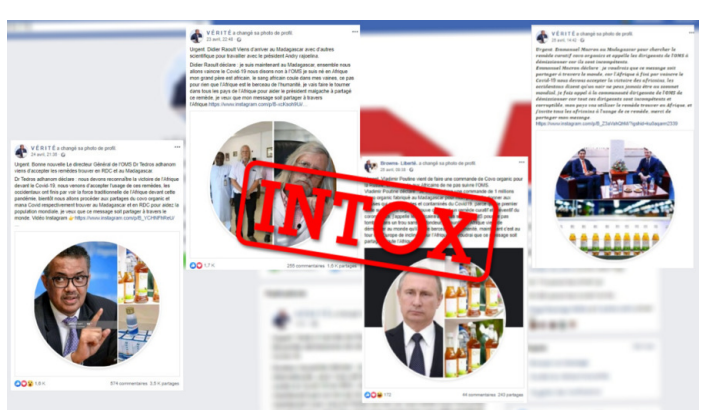

Fig. 1 A 20-year-old Congolese student posts a disinformation post about New Coronary Pneumonia

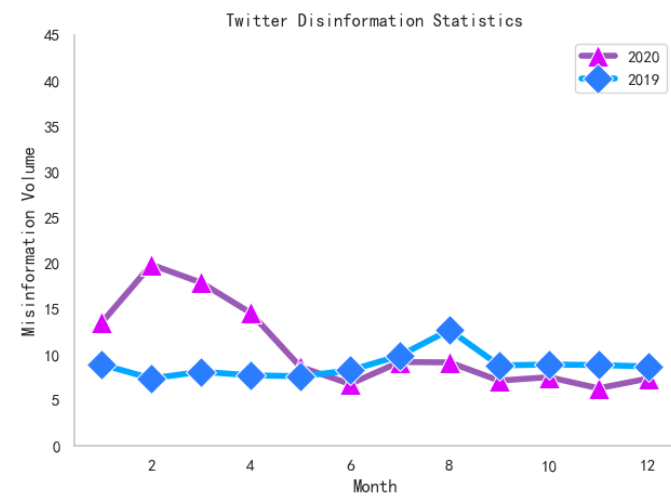

Fig. 2 Comparison of inaccurate information statistics on Twitter in 2020 vs. 2019

million(Facebook, Community Standards Enforcement Report, November 2019 Edition). According to research by the German Marshall Fund[1], misinformation on Twitter and Facebook increased rather than decreased in 2020, as is shown in Figure 1. For example, a 20-year-old Congolese student gained 60,000 followers by posting a disinformation post about New Coronary Pneumonia(France 24, The Observers). Dissemination of information through the internet is not only of low-cost and fastspeed, but also has a wide impact, which is why it can easily become a bad means of spreading rumors and result in certain negative effects[2]. Taking the official data released by Weibo Refutes Rumors in 2019 and 2020 as an example, in Figure 2, the annual average reports of false information in 2019 is around 88,500, while that in 2020 is about 106,850(Annual Report on the Work of Weibo Disinformation, 2019, 2020). It is easy to see that the number of rumors containing false information is still slowly increasing. The detection of false information[3], i.e. rumor detection, has therefore become one of the most urgent priorities of online purification and attracted wide social attention.

With the development and application of artificial intelligence, there has been an increase in the methods of rumor detection, including those based on classifier-, propagation structure, open-source evidence, information source, etc. However, existing 
efforts in automatic rumor detection have mainly focused on text analysis and feature extraction based on user-posted comments, such as extracting keywords from a text by Dadvar[4] and extracting text features from the perspective of gramma. Depth sequences (Lin et al.)[5] is one of such practices. Although the detection performance of the methods used in these models can achieve quite satisfying results, many challenges still remain, especially those concerning user interaction features and global semantic features.

Our work aims to detect online rumors by studying user comments and potential user interactions on social media. Furthermore, we achieve this goal by constructing Multi-Attention Neural Interaction Network (MANIN), which consists of three main modules to learn about the interactions among messages in social networks. We use BERT, which is based on 12 transform layers, to enhance global semantic information features so as to capture the semantic and syntactic cues of disinformation. We create a post-comment co-attention neural network to learn about the text-level interactions between the posted texts and the comments. In addition, we also learn about the propagation representations among users by extracting characteristics, which describe potential interactions.

The contribution of our paper is in two main areas, as follows:

- We design a novel model, the Multi-Attention Neural Interaction Network (MANIN), which better learns the common correlations between the original tweets and comments, as well as the potential user interaction representations.

- Experiments on a real tweet dataset show that our model outperforms other existing models and detects rumors satisfactorily in the early stages.

\section{Related Work}

In this section, relevant research can be divided into human-based detection, machine learning-based method, and deep learning method. Manual rumor detection is still the main detection method on social networking platforms, in which suspicious information is handed over to experts, experienced editors, or third-party official platforms in relevant field to verify the authenticity of the information. For instance, Facebook[6] is still using the manual rumor detection method, which is very effective in results but slow in speed. Machine learning-based approach focuses on selecting and extracting features from data that effectively characterize it, training classification models, and making predictions. Feature-based approaches mainly include text features, user features, and communication features. The text feature-based[7] approach focuses on rumor identification by using a variety of texts, including the original text, comments, forwarded content, etc. These various text features are captured by recurrent neural networks. For example, relevant semantic features of text sequences are captured by recurrent neural networks, which preserve the linguistic features among texts, and Ma et al.[8] used this to better study the representation of texts. Liao et al.[9] also achieved desirable results by mining the potential features of texts in terms of time segments. The user-based features usually include user personal information features (Sun[10]), user comment relationship features (Ma[11]), and user behavior features (Guo[12]), etc. Whereas deep learning has strong feature-studying capacity. Chen[13] 
proposed a deep attention model based on recurrent neural networks in 2017, which delves soft attention into recurrent neural networks to capture the context of relevant posts changing over time. DEFEND(Shu et al.[14]) combines the clocking effect of responding to comments and the correlation between message contents and comments, and uses a focus mechanism to provide interpretability of the models.

In comparison to our work, we also use a fusion of multiple features and incorporate a multi-attention mechanism to better enhance model detection in a number of ways and use the model to provide evidence of validity.

\section{Problem Statement}

Social rumor detection aims to identify whether a twitter text on a social networking platform is a rumor via each event's relevant information, including the source posts, user comments, and potential propagation relationship. Let $\mathrm{P}=\left\{p_{1}, p_{2}, p_{3}, \ldots, p_{N}\right\}$ be a corpus of source posts on social media, $\mathrm{C}=\left\{c_{1}, c_{2}, c_{3}, \ldots, c_{M}\right\}$ be a corpus of user comments, and $\mathrm{U}=\left\{u_{1}, u_{2}, u_{3}, \ldots, u_{T}\right\}$ be a corpus of social interaction users. Each source post contains the post text and subsequent comments. We consider the social rumor detection as a binary classification task, in which each tweet is associated with a binary label $y_{k}=\{0,1\}$ to represent is authenticity, where $y_{k}=0$ denotes it is a rumor, and $y_{k}=1$ indicates it is not a rumor.

Given a source posts $p_{i}$, a set of interrelated comments $\mathrm{C}$ and a user interaction graph $G_{u u}$, the purpose is to make predictions about rumor detection by studying the detection function $\mathrm{f}: \mathrm{f}\left(\mathrm{P}, \mathrm{C}, G_{u u}\right) \rightarrow(\hat{y})$. Furthermore, it could maximize the accuracy of predictions with explainable user comments.

\section{The Proposed Model}

In this work, the details of the model proposed by the author is described, which incorporates the multi-attention mechanism, including Bidirectional Encoder Representations from Transformers, co-attention mechanism and graph attention network, to effectively detect social network rumors. As is shown in Figure 3, this model consists of four major components. The first part is a text encoder(including source posts and user comments), which employs the pre-trained BERT model to extract the text features. The second part is a co-attention mechanism, which learns about the interactions between the posts and the comments by studying the two. The third part is a propagation representation, which extracts the underlying characteristics of user interactions via GAT. The final part is making predictions based on all the representations obtained above.

\subsection{Text Encoding}

While Word2Vec[15] and Glove[16] are two good methods for calculating the semantic similarity of words, BERT[17] is superior to both of them. One interesting thing about BERT is that it calculates not only word and tag embedding, but also 


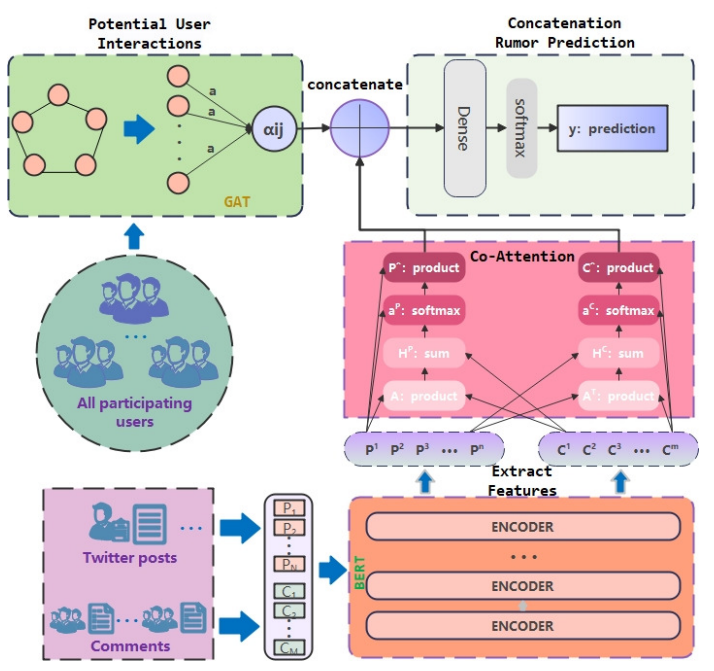

Fig. 3 The structure of the proposed MANIN model

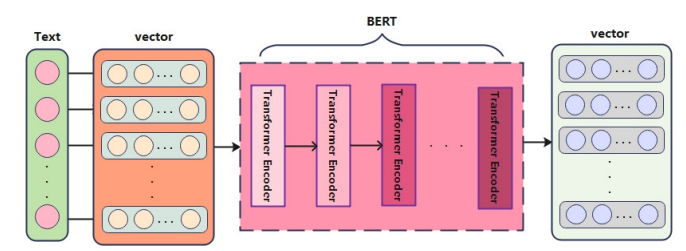

Fig. 4 An structure of text embedding model

sentence embedding, as a way to distinguish the position of a sentence and a word in a given sequence. Another interesting thing about BERT is that it hides $15 \%$ of the words and uses the positional embedding to process or infer them, thus making the studying more efficient. Therefore, we utilize pre-trained BERT as a text encoder to encode source posts and user comments, as a way to effectively obtain the contextual representation of a sentence.

Figure 4 illustrates the basic structure of text embedding via BERT model. To obtain a semantically rich representation of the text, we first represent the words in the text as raw vectors, then fuse the vector representation with the full-text semantic information, and finally perform a series of complex transformations through BERT to output the semantic representation of the text. In this work, the BERT part consists of 12 layers(https://github.com/google-research/bert) of transformers[18] to generate the final embedding. Each layer consists of only the transformer encoder, which encodes the input data (generates the embedding). 


\subsection{Co-attention Mechanism}

To better study the mutual interaction between posts and comments as a way to find valid evidence to confirm if a message is false statement, we propose to utilize a coattention mechanism[19] to learn about the semantic word-level correlation between the posts and the comments. In other words, we intend to simultaneously learn about and generate attention the weights for words on posts and comments. To put it specifically, we build a model to reflect the mutual influence between the feature matrix of words in the posts (i.e., $\mathrm{P}=\left[p^{1}, p^{2}, p^{3}, \ldots, p^{n}\right]$ ) and that of the comments (i.e., $\mathrm{C}=[$ $\left.\left.c^{1}, c^{2}, c^{3}, \ldots, c^{m}\right]\right)$.

By extending the presentation of co-attention, we first calculate the affinity matrix $\mathbf{A} \in \mathbb{R}^{m \times n}: \mathbf{A}=\tanh \left(C^{T} W_{c p} \mathrm{P}\right)$, where $W_{c p}$ is a matrix of learnable parameters. This affinity matrix $\mathbf{A}$ could be used to transform the comment attention space to the post text attention space, and vice versa for $\mathbf{A}^{T}$. By treating the proximity matrix as a feature, we could study it to predict the source and interaction attention maps, given by:

$$
\begin{aligned}
& \mathbf{H}^{c}=\tanh \left(\mathbf{W}_{c} \mathbf{C}+\left(\mathbf{W}_{p} \mathbf{P}\right) \mathbf{A}^{T}\right) \\
& \mathbf{H}^{p}=\tanh \left(\mathbf{W}_{p} \mathbf{P}+\left(\mathbf{W}_{c} \mathbf{C}\right) \mathbf{A}\right)
\end{aligned}
$$

where $\mathbf{W}_{c}, \mathbf{W}_{p}$ are the learnable parameters. Then, the attention weights of posted text and comments can be generated by:

$$
\begin{aligned}
& \mathbf{a}^{c}=\operatorname{softmax}\left(\mathbf{w}_{h c}^{T} \mathbf{H}^{c}\right) \\
& \mathbf{a}^{p}=\operatorname{softmax}\left(\mathbf{w}_{h p}^{T} \mathbf{H}^{p}\right)
\end{aligned}
$$

where $\mathbf{w}_{h c}, \mathbf{w}_{h p}$ are vectors of learnable weight parameters. $\mathbf{a}^{c}, \mathbf{a}^{p}$ are the vectors of attention probabilities for each word in the posts and comments. Ultimately, according to the attention weights obtained above, we could calculate the attention vectors of the posts and comment by using the weighted sum of the derived attention weights, as is shown below:

$$
\hat{\mathbf{c}}=\sum_{i=1}^{M} \mathbf{a}_{i}^{c} \mathbf{c}^{i}, \hat{\mathbf{p}}=\sum_{i=1}^{N} \mathbf{a}_{i}^{p} \mathbf{p}^{i}
$$

where $\hat{\mathbf{c}}, \hat{\mathbf{p}}$ are the calculated co-attention features vectors for the posts and comments respectively.

\subsection{Interaction Extractors}

To research and learn about the potential relationships between posting and commenting users, we use diagrams to model the interactions between users. We utilize multilayer neural networks that operate on graph data based on the layers of the graph attention neural networks (GAT[20]). Based on the feature embedding of fused nodes, GAT uses an attention mechanism to do aggregation operations on neighbor nodes to achieve the adaptive assignment of weights to different neighbors and to better learn about potential interactions[21] between users.

Therefore, let $\mathrm{H}=\left(h_{1}, h_{2}, h_{3}, \ldots, h_{n},\right) \in \mathbb{R}^{n \times u}$ be the interaction vectors for users participating in all posts, where $\mathrm{n}$ is the number of all comments and $\mathrm{p}$ is the number 
of all users participating in the interactions. Then, let the model learn about the representation relation of the interaction from the graph $G=(H, A)$, where $A \in \mathbb{R}^{n \times n}$ encodes the pairwise relationships between users. After that, we learn about the weight among nodes in the graph utilizing a self-attention approach. Given a node pair $\left(h_{i}\right.$, $h_{j}$ ) in the graph, the self-attention mechanism $f$ could be used to learn the coefficient of attention, which represents the importance of $\mathbf{e}_{i, j}$. The coefficient of attention could be calculated by the following formula:

$$
\mathbf{e}_{i, j}=f\left(W h_{i}, W h_{j}\right)
$$

where $W$ represents the weight matrix of a sharing linear transformation. And $f$ could be realized by a single-layer feed-forward neural network which is parameterized by a weight vector a and uses LeakyReLU as the activation function. Then, we utilize the softmax function to normalize them and obtain the coefficients $\alpha_{i, j}$ as follows:

$$
\alpha_{i, j}=\operatorname{softmax}\left(e_{i, j}\right)=\frac{\exp \left(\sigma\left(\mathbf{a}^{T} \cdot\left[W h_{i} \| W h_{j}\right]\right)\right)}{\sum_{k \in \mathscr{N}_{i}} \exp \left(\sigma\left(\mathbf{a}^{T} \cdot\left[W h_{i} \| W h_{k}\right]\right)\right)}
$$

here $\sigma$ represents the activation function, we chose LeakyRelu as the activation function for our experiment. $\|$ indicates the concatenation operation, a denotes the weight vector of the neural network and ${ }^{T}$ represents the transpose operation, and $\mathscr{N}_{i}$ indicates the set which contains a node $i$ and its neighbors. After that, we aggregate the neighbor representations of a node $i$ in the graph and their corresponding coefficients to update the embedding of a node $\mathrm{i}$, given by:

$$
\mathbf{h}_{i}^{\prime}=\sigma\left(\sum_{j \in \mathscr{N}_{i}} \alpha_{i j} W h_{j}\right)
$$

where $\mathbf{h}_{i}^{\prime}$ indicates the new feature for each vertex i (incorporating neighborhood information). $\sigma(\cdot)$ is a nonlinear function, $W$ represents the same weight matrix as Equation 3, and $\mathscr{N}_{i}$ indicates the set which contains a node i and its neighbors. Eventually, we employ self-attention and extend it to multi-headed attention to learn about how to embed better. The final output representation can be obtained by concatenating the results of the above conversion as follows:

$$
\mathbf{h}_{i}^{\prime}(K)=\|_{k=1}^{K} \sigma\left(\sum_{j \in \mathscr{N}_{i}} \alpha_{i j}^{k} W^{k} h_{j}\right)
$$

where $\alpha_{i j}^{k}$ represents the normalized attention coefficients obtained by the k-th attention mechanism $\left(f^{k}\right)$, and $W^{k}$ represents the corresponding weight matrix of input linear transformation, and $\|$ indicates the concatenation operation.

\subsection{Making Predictions}

We mainly connect interactive co-attention feature vectors $\hat{\mathbf{p}}$ and $\hat{\mathbf{c}}$, as well as user interaction representation feature vector $\hat{\mathbf{h}}$ to implement rumor detection. Let $f=[\hat{\mathbf{p}}$, 
Table 1 Attributes of two datasets

\begin{tabular}{lll}
\hline Statistic & PHEME2016 & PHEME2018 \\
\hline \#rumors & 62 & 2091 \\
\#nonrumors & 235 & 3650 \\
\#source tweets & 297 & 5741 \\
\#comments & 2509 & 53474 \\
\#users & 2806 & 59215 \\
\hline
\end{tabular}

$\hat{\mathbf{c}}, \hat{\mathbf{h}}]$ which is then fed into a multi-layer feedforward neural network to generate a prediction label. It can be derived as follows:

$$
\hat{\mathbf{y}}=\sigma\left([\hat{\mathbf{p}}, \hat{\mathbf{c}}, \hat{\mathbf{h}}] \mathbf{W}_{f}+\mathbf{b}_{f}\right)
$$

where $\sigma$ is the sigmoid function, $\mathbf{W}_{f}$ is the matrix of learnable parameters, and $\mathbf{b}_{f}$ is the bias term. Our aim is to minimize the cross-entropy loss function:

$$
\mathscr{L}(\Theta)=-y \log (\hat{y})-(1-y) \log (1-\hat{y})
$$

where $\Theta$ denotes all learnable parameters in the entire network. We utilize the Adam optimizer, which combines the advantages of AdaGrad (for sparse gradients) and RMSProp (for online data) and does not require the setting of step decays, to learn about these parameters.

\section{Experiments}

In this section, we aim to address this issue by conducting experiments and analyses in three main areas: 1)How effective is rumor detection? 2)Is rumor detection effective in the early stages? 3)What is the result of the ablation analysis of the model?

\subsection{Datasets Description and Settings}

We conducted experiments using the publicly available datasets PHEME2016[22] and PHEME2018[23], which contain data such as tweets commenting users' posts and users' information. Each source post in the dataset was marked as either 0 or 1 , with 0 being "false" and 1 being "true". As shown in Table 1, in general, the PHEME dataset from Twitter is based on real event themes and it contains fewer rumors than non-rumors.

To compare different models fairly, we use four metrics: accuracy, recall, precision and F1 score, to comment on their performance. Our framework is mainly implemented by taking TensorFlow[24] as the back-end keras[25]. We select the best parameter settings based on the performance of the verification set and evaluate our framework performance on the test set. 
Table 2 The main performance comparison in four metrics for rumor detection on two datasets

\begin{tabular}{lllllllll}
\hline & \multicolumn{3}{c}{ PHEME2016 } & \multicolumn{3}{c}{ PHEME2018 } \\
\hline Method & Accuracy & F1-score & Precision & Recall & Accuracy & F1-score & Precision & Recall \\
\hline CONcISE & 0.589 & 0.301 & 0.369 & 0.255 & 0.598 & 0.666 & 0.631 & 0.706 \\
LSTM & 0.656 & 0.524 & 0.507 & 0.542 & 0.649 & 0.765 & 0.747 & 0.784 \\
GRU & 0.666 & 0.550 & 0.592 & 0.514 & 0.609 & 0.748 & 0.707 & 0.795 \\
GRU-2 & 0.712 & 0.671 & 0.753 & 0.605 & 0.638 & 0.775 & 0.744 & 0.807 \\
MTB & 0.726 & 0.687 & $\mathbf{0 . 7 1 7}$ & 0.659 & 0.770 & 0.866 & 0.825 & 0.912 \\
\hline MANIN-A & 0.742 & 0.666 & 0.758 & 0.594 & 0.746 & 0.876 & 0.867 & 0.886 \\
MANIN & $\mathbf{0 . 7 9 2}$ & $\mathbf{0 . 7 5 1}$ & 0.710 & $\mathbf{0 . 7 9 7}$ & $\mathbf{0 . 8 1 6}$ & $\mathbf{0 . 9 1 4}$ & $\mathbf{0 . 9 0 8}$ & $\mathbf{0 . 9 2 1}$ \\
\hline
\end{tabular}

\subsection{Rumor Detection Performance}

Experiments were conducted on several methods and the model proposed in this study by employing PHEME dataset. The main results of the rumor detection experiments are shown in Table 2 . We can clearly find that our model significantly outperforms other competing approaches in terms of metrics. These good experimental results show that the model framework proposed by us could better capture the semantic relations and effective features in the textual content and that this can be of great help in rumor detection. In addition, these results also imply that 1) the significant performance of word embeddings with pre-trained BERT is shown, 2) the co-attention mechanism is of considerable importance in the model, and 3) the potential user interactions also provide effective help.

\subsection{Early Rumor Detection}

In order to contain rumor outbreaks in the early stage, we need to investigate whether the model can accurately detect them in their early stages. To put it specifically, we want to understand how well a model performs when there are only partially observed comments. Using accuracy as the evaluation metrics, it is clear from the results in Figure 5 that, the model achieves good performance when the number of comments is small. In contrast, the early detection performance of the other methods is inferior to our proposed model. Overall, we demonstrate that our model is still quite accurate in detecting rumors at the early stage, which is crucial in defending against rumor outbreaks.

\subsection{Ablation Study}

We show the importance of each component by removing some components from the complete model. Where 'All' indicates that all components of the model are used. Then, by progressively removing the BERT encoder, the user-based GAT representation, and the post-based and comment-based co-attention, we generated "-A", "-B", "-G", "-BG" and "-AG" respectively. The results are shown in Figure 6, where we 


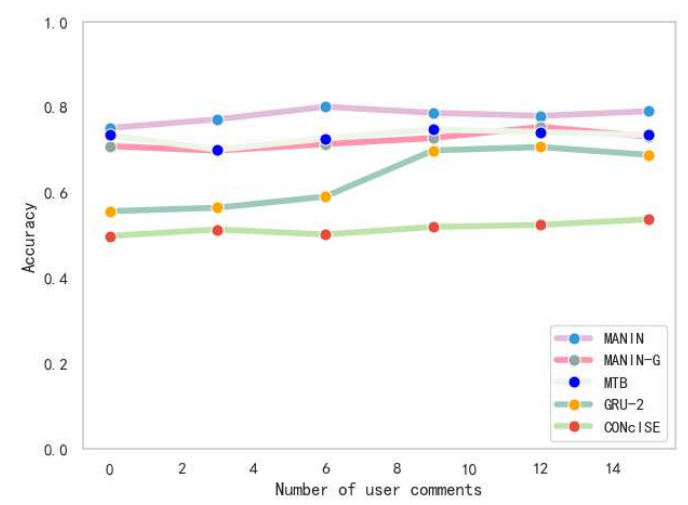

Fig. 5 Accuracy of early rumor detection at different checkpoints

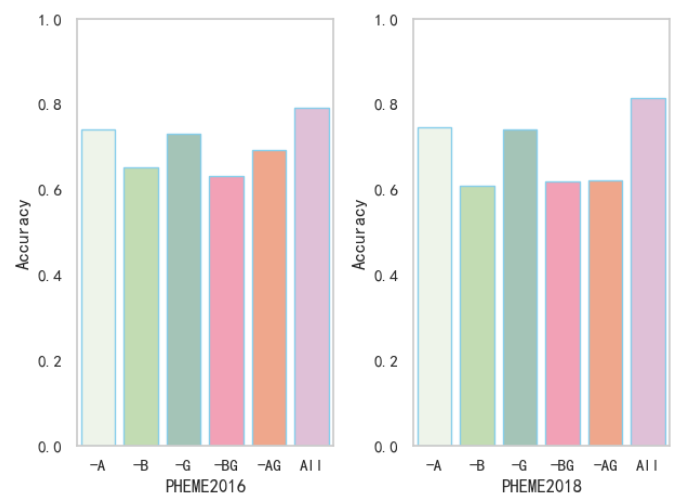

Fig. 6 MANIN ablation analysis in Accuracy

can find that each component does play its role and has some effect on the overall model, especially the co-attention ("-A"), without which it would be difficult to learn about the common representation of posts and comments and accuracy would be significantly reduced.

\subsection{Case Study}

These cases are presented in Figure 7. We can find that MANIN can give different attention weights to user comments according to their relevance to the original posts, and then rank them from highest to lowest according to their attention weights. For example, if the original post is: "Unconfirmed reports claim that Michael Essien has contracted Ebola.", we can list the top three comments out of the 15 comments used to explain this original post, which makes the model very explanatory. Overall, MANIN could not only accurately detect rumors, but also highlight key evidential comments as explanations. 


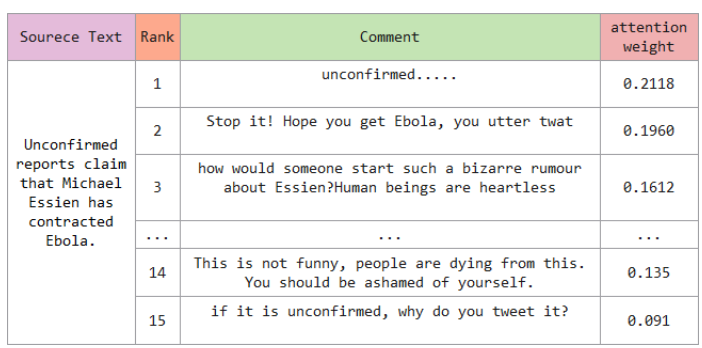

Fig. 7 Relevance ranking of comments by MANIN

\section{Conclusion and Future Work}

In recent years, rumor detection on social networks has turned out to be an important issue which draws increasing attention. To better address this problem, this study proposes a rumor detection model based on a multi-attention module. It employs a self-attention-based BERT encoder to enhance the global semantic features and to better learn about the representation of features through the co-attention of posts and comments and the potential interactions between users. The experimental results show that the model has better detection performance and effective recognition. Such results encourage our to incorporate more heterogeneous information in future researches, including individual user features and propagation features, as well as image features that introduce posting by users, as a way to verify if our model performance could be further enhanced.

Acknowledgements This work is supported by the Innovation Fund of Postgraduate, Xihua University(YCJJ2021028).

\section{References}

1. Kornbluh K, Goldstein A, Weiner E. New Study by Digital New Deal Finds Engagement with Deceptive Outlets Higher on Facebook Today Than Run-up to 2016 Election. GMF The German Marshall Fund of the United States. Viitattu 16.12. 2020[J]. 2020

2. Barua Z, Barua S, Aktar S, et al. Effects of misinformation on COVID-19 individual responses and recommendations for resilience of disastrous consequences of misinformation[J]. Progress in Disaster Science, 2020, 8: 100119.

3. Zannettou S, Sirivianos M, Blackburn J, et al. The web of false information: Rumors, fake news, hoaxes, clickbait, and various other shenanigans[J]. Journal of Data and Information Quality (JDIQ), 2019, 11(3): 1-37.https: / / arxiv.org/pdf/1804.03461.pdf

4. Dadvar M, Trieschnigg D, Ordelman R, et al. Improving cyberbullying detection with user context[C]//European Conference on Information Retrieval. Springer, Berlin, Heidelberg, 2013: 693-696.

5. Lin D, Ma B, Cao D, et al. Chinese microblog rumor detection based on deep sequence context[J]. Concurrency and Computation: Practice and Experience, 2019, 31(23): e4508.

6. Kochkina E, Liakata M, Zubiaga A. Pheme dataset for rumour detection and veracity classification[J]. figshare, Jun, 2018

7. Castillo C, Mendoza M, Poblete B. Information credibility on twitter[C]//Proceedings of the 20th international conference on World wide web. 2011: 675-684.https: / / d . a cm. org/doi/abs/10. $1145 / 1963405.1963500$

8. Ma J, Gao W, Mitra P, et al. Detecting rumors from microblogs with recurrent neural networks[J]. 2016. 
9. Liao X, Huang Z, Yang D, et al. Rumor detection in social media based on a hierarchical attention network[J]. SCIENTIA SINICA Informationis, 2018, 48(11): 1558-1574.

10. Sun S, Liu H, He J, et al. Detecting event rumors on sina weibo automatically[C]//Asia-Pacific web conference. Springer, Berlin, Heidelberg, 2013: 120-131.

11. Ma J, Gao W, Wong K F. Rumor detection on twitter with tree-structured recursive neural networks[C]. Association for Computational Linguistics, 2018.

12. Guo H, Cao J, Zhang $\mathrm{Y}$, et al. Rumor detection with hierarchical social attention network[C]//Proceedings of the 27th ACM International Conference on Information and Knowledge Management. 2018: 943-951.

13. Chen T, Li X, Yin H, et al. Call attention to rumors: Deep attention based recurrent neural networks for early rumor detection[C]//Pacific-Asia conference on knowledge discovery and data mining. Springer, Cham, 2018: 40-52.

14. Shu K, Cui L, Wang S, et al. defend: Explainable fake news detection[C]//Proceedings of the 25th ACM SIGKDD international conference on knowledge discovery \& data mining. 2019: 395405.https://dl.acm.org/doi/abs/10.1145/3292500.3330935

15. Mikolov T, Chen K, Corrado G, et al. Efficient estimation of word representations in vector space[J]. arXiv preprint arXiv:1301.3781,2013.https://arxiv.org/abs/1301.3781

16. Pennington J, Socher R, Manning C D. Glove: Global vectors for word representation[C]/Proceedings of the 2014 conference on empirical methods in natural language processing (EMNLP). 2014: 1532 1543.https: / / aclanthology.org/D14-1162.pdf

17. Devlin J, Chang M W, Lee K, et al. Bert: Pre-training of deep bidirectional transformers for language understanding[J]. arXiv preprint arXiv:1810.04805, 2018.https://arxiv.org/abs/ 1810.04805

18. Wolf T, Debut L, Sanh V, et al. Huggingface's transformers: State-of-the-art natural language processing[J]. arXiv preprint arXiv:1910.03771, 2019.https: / / arxiv.org/abs/1910.03771

19. Lu J, Yang J, Batra D, et al. Hierarchical question-image co-attention for visual question answering[J]. Advances in neural information processing systems, 2016, 29: 289-297.

20. Veličković P, Cucurull G, Casanova A, et al. Graph attention networks[J]. arXiv preprint arXiv:1710.10903, 2017.https://arxiv.org/abs/1710.10903

21. Huang Q, Yu J, Wu J, et al. Heterogeneous graph attention networks for early detection of rumors on twitter[C]//2020 International Joint Conference on Neural Networks (IJCNN). IEEE, 2020: 1-8.https : //ieeexplore.ieee.org/abstract/document/9207582/

22. Zubiaga A, Liakata M, Procter R, et al. Analysing how people orient to and spread rumours in social media by looking at conversational threads[J]. PloS one, 2016, 11(3): e0150989.https: //journals.plos.org/plosone/article?id=10.1371/journal.pone.0150989

23. Kochkina E, Liakata M, Zubiaga A. Pheme dataset for rumour detection and veracity classification[J]. figshare, Jun, 2018.

24. Sergeev A, Del Balso M. Horovod: fast and easy distributed deep learning in TensorFlow[J]. arXiv preprint arXiv:1802.05799, 2018.https: / / arxiv.org/abs/1802.05799

25. Gulli A, Pal S. Deep learning with Keras[M]. Packt Publishing Ltd, 2017. 